OPEN ACCESS

\section{Check for updates}

For numbered affiliations see end of the article.

Correspondence to: $\mathrm{BX}$ xibo2007@126.com

(ORCID 0000-0003-0491-5585) Additional material is published online only. To view please visit the journal online.

Cite this as: $B M / 2020 ; 370: \mathrm{m} 2031$ http://dx.doi.org/10.1136/bmi.m2031

Accepted: 21 April 2020

\title{
Recommended physical activity and all cause and cause specific mortality in US adults: prospective cohort study
}

\author{
Min Zhao, ${ }^{1,2}$ Sreenivas P Veeranki, ${ }^{3,4}$ Costan G Magnussen, ${ }^{5,6,7}$ Bo Xi ${ }^{8}$
}

\begin{abstract}
OBJECTIVE

To determine the association between recommended physical activity according to the 2018 physical activity guidelines for Americans and all cause and cause specific mortality using a nationally representative sample of US adults.

DESIGN

Population based cohort study.

SETTING

National Health Interview Survey (1997-2014) with

linkage to the National Death Index records to 31

December 2015.

PARTICIPANTS

479856 adults aged 18 years or older.

EXPOSURES

Participant self-reports of the amount of leisure time spent in aerobic physical activity and muscle strengthening activity each week were combined and categorised into four groups: insufficient activity, aerobic activity only, muscle strengthening only, and both aerobic and muscle strengthening activities according to the physical activity guidelines.
\end{abstract}

\section{MAIN OUTCOME MEASURES}

All cause mortality and cause specific mortality (cardiovascular disease, cancer, chronic lower respiratory tract diseases, accidents and injuries, Alzheimer's disease, diabetes mellitus, influenza and pneumonia, and nephritis, nephrotic syndrome, or nephrosis) obtained from the National Death Index records.

\section{RESULTS}

During a median follow-up of 8.75 years, 59819 adults died from all causes, 13509 from cardiovascular disease, 14375 from cancer, 3188 from chronic lower respiratory tract diseases, 2477 from accidents and injuries, 1470 from Alzheimer's disease, 1803 from diabetes mellitus, 1135 from

\section{WHAT IS ALREADY KNOWN ON THIS TOPIC}

Only four studies have assessed the association between meeting the physical activity guidelines and mortality due to all causes, cardiovascular disease, or cancer, with inconsistent results

No studies have assessed the association with other cause specific mortality (eg, deaths due to Alzheimer's disease or diabetes mellitus)

\section{WHAT THIS STUDY ADDS}

Adults who perform physical activity at levels recommended by the 2018 physical activity guidelines for Americans show a greatly reduced risk of all cause and cause specific mortality

The beneficial effects on mortality risk are largely comparable between two intensities of aerobic activity each week ( $\geq 150$ minutes of light to moderate intensity $v \geq 75$ minutes of vigorous intensity) influenza and pneumonia, and 1129 from nephritis, nephrotic syndrome, or nephrosis. Compared with those who did not meet the physical activity guidelines ( $n=268193)$, those who engaged in recommended muscle strengthening activity ( $n=21428$; hazard ratio $0.89,95 \%$ confidence interval 0.85 to 0.94$)$ or aerobic activity $(n=113851 ; 0.71$, 0.69 to 0.72 ) were found to be at reduced risk of all cause mortality; and even larger survival benefits were found in those engaged in both activities ( $n=76384$; $0.60,0.57$ to 0.62 ). In addition, similar patterns were reported for cause specific mortality from cardiovascular disease, cancer, and chronic lower respiratory tract diseases.

\section{CONCLUSIONS}

Adults who engage in leisure time aerobic and muscle strengthening activities at levels recommended by the 2018 physical activity guidelines for Americans show greatly reduced risk of all cause and cause specific mortality. These data suggest that the physical activity levels recommended in the guidelines are associated with important survival benefits.

\section{Introduction}

Inadequate physical activity is a worldwide public health problem, with considerable healthcare and economic burden. Physical inactivity is estimated to be responsible for $6-10 \%$ of the global burden of major chronic non-communicable diseases and 9\% of premature deaths (based on the proportion of inactive people among cases and the relative risk of disease when inactive people were compared with active people) in $2008^{1}$ and attributed to a total cost of $\$ 53.8 \mathrm{bn}$ (£43.8bn; €49.0bn) to healthcare systems worldwide in 2013 . $^{2}$ Among all countries, the United States has the highest economic burden, of about $\$ 24.7 \mathrm{bn}$ in healthcare costs (accounting for 45.9\% of global healthcare costs) from physical inactivity. ${ }^{2}$ Evidence is growing for the protective effect of higher physical activity levels in preventing many chronic diseases (such as cardiovascular disease, type 2 diabetes, and cancer) ${ }^{3}$ and in reducing all cause mortality. ${ }^{4}$ Therefore, engaging in physical activity is important to prevent or limit morbidity and mortality from future chronic disease and thereby reduce healthcare expenditure and losses to productivity.

The 2018 physical activity guidelines for Americans, ${ }^{5}$ consistent with the 2008 guidelines, ${ }^{6}$ recommends that adults should engage in at least 150 minutes of moderate intensity aerobic physical activity or at least 75 minutes of vigorous intensity aerobic physical activity each week, or an equivalent combination of both. Furthermore, adults should engage in muscle strengthening activities of moderate or greater intensity on two days or more each week. 
Evidence as to whether these recommendations are met is associated with reduced mortality among US adults is, however, lacking and inconsistent. ${ }^{7-10}$ For example, two studies in the US and one in the United Kingdom found a statistically significant reduction in all cause mortality for those who met recommended physical activity levels, ${ }^{7810}$ whereas a study in the US did not. ${ }^{9}$ Furthermore, only two studies have assessed the risk of cardiovascular disease specific and cancer specific mortality attributed to physical inactivity, again with inconsistent results. ${ }^{710}$ Additionally, no studies have examined the association between meeting sufficient physical activity and other cause specific mortality, such as deaths due to Alzheimer's disease or diabetes. Given the protective effect of physical activity on general health, inconsistent previous findings, and the existence of new (2018) physical activity guidelines, we determined the association between meeting recommended physical activity levels according to the 2018 guidelines and all cause and cause specific mortality using pooled data of a nationally representative sample of US adults.

\section{Methods}

Study population

The study included data from the National Health Interview Survey (NHIS), an ongoing national yearly cross sectional household interview survey conducted since 1957 by the US Centers for Disease Control and Prevention and the National Center for Health Statistics through the US Census Bureau. Potential participants are selected using a stratified, multistage probability design and are representative of the civilian US population living in the community. Basic health information is collected for all household members, and additional information, including health status, health behaviours, and healthcare utilisation, is obtained from one randomly sampled adult in each family. Detailed information on the design, methodology, and weighting is described elsewhere. ${ }^{11}$

This study included representative adult data from each household collected during 1997-2014 (all participants across the surveys were unique), with linkage to the National Death Index to 31 December 2015. Since the NHIS questionnaires underwent a major revision in 1997, we did not include earlier surveys. A total of 529363 participants aged 18 years or older met the enrolment criteria and were included in this study. Among these, 49507 were excluded because of pregnancy $(n=6175)$, missing data on aerobic physical activity or muscle strengthening activity $(n=16668)$, and missing data on potential covariates (personal variables, lifestyle factors, or chronic health conditions; $\mathrm{n}=26664$ ), resulting in a final analytical sample of 479856 adults. Participants in the analytical sample ranged from 31959 in 1997 to 33035 in 2014. Reasons for missing data on aerobic physical activity and muscle strengthening activity, as well as potential covariates, were that participants were not sure, did not know the answer, or refused to respond.

\section{Aerobic and muscle strengthening activities}

At each survey from 1997 to 2014, study participants self-reported the frequency and duration of leisure time aerobic activity, as well as the frequency of muscle strengthening activity in a usual day, week, month, and year over the past year. Frequency was assessed as aerobic activity for at least 10 minutes (according to previous guidelines ${ }^{6}$ ) and categorised into light to moderate intensity (equal to a metabolic equivalent of task (MET) value of <6; such as gardening, brisk walking, and dancing) that causes only light sweating or a slight to moderate increase in breathing or heart rate, and vigorous intensity (equal to a MET value of $\geq 6$; such as running, faster cycling, and competitive sports) that causes heavy sweating or much increased breathing or heart rate. To measure leisure time aerobic physical activity, defined in minutes/week, we multiplied frequency (times/week) by duration of activity (minutes/time). Muscle strengthening activity was defined by frequency (times/week) using self-reported responses to the question "How often do you do physical activities specifically designed to strengthen your muscles, such as lifting weights or doing calisthenics?" Following the 2018 guidelines, ${ }^{5}$ for aerobic physical activity, we categorised the study participants into two groups: recommended activity ( $\geq 150$ minutes of light to moderate intensity activity each week, or $\geq 75$ minutes of vigorous intensity activity, or greater than or equal to an equivalent combination) and insufficient activity ( $<150$ minutes of light to moderate intensity activity each week and $<75$ minutes of vigorous intensity activity, and less than an equivalent combination). Muscle strengthening activity was also categorised into two groups: recommended ( $\geq 2$ times/week) and insufficient ( $<2$ times/week). Using the two categories each for aerobic physical activity and muscle strengthening activity, we classified participants into one of four groups depending on whether they met each of the recommended guidelines: insufficient activity (insufficient aerobic and muscle strengthening activities), aerobic physical activity only (recommended aerobic activity and insufficient muscle strengthening activity), muscle strengthening only (insufficient aerobic activity and recommended muscle strengthening activity), and both (recommended aerobic and muscle strengthening activities).

\section{Mortality}

We linked eligible participants in the NHIS from 1997 to 2014 to the National Death Index records up to 31 December 2015. To determine mortality status of the NHIS participants, we used a probabilistic record matching method with the National Death Index records. ${ }^{12}$ The accuracy of information on all cause and cause specific mortality in the National Death Index records has been validated in previous studies. ${ }^{1314}$ Follow-up duration was determined as the time from participation in the NHIS to death for decedents or to the censoring date (31 December 2015) for survivors. Cause of death was coded using ICD-10 (international classification of diseases, 10th 
revision). ${ }^{15}$ The main outcome was all cause and cause specific mortality (cardiovascular disease, cancer, chronic lower respiratory tract diseases, accidents and injuries, Alzheimer's disease, diabetes, influenza and pneumonia, and nephritis, nephrotic syndrome, or nephrosis). See box 1 for the ICD-10 codes.

\section{Study covariates}

Several participant characteristics were included as covariates to reduce confounding: personal variables (age, sex, race/ethnicity (non-Hispanic white, nonHispanic black, Hispanic, and others), education (less than high school, high school, and more than high school), and marital status (married; divorced, separated, or widowed; and never married)); lifestyle variables (body mass index, smoking status, and alcohol intake); and chronic health conditions (hypertension, heart disease, stroke, cancer, and diabetes) defined using self-reported responses of participants about whether a doctor or other health professional had ever informed them that they had a specific chronic condition. Body mass index was calculated using self-reported height and weight, and categorised into normal weight $\left(<25 \mathrm{~kg} / \mathrm{m}^{2}\right)$, overweight $(25-<30)$, and obese $(\geq 30)$. Smoking status was defined as never, former, and current smoker using self-reported responses to the questions: "Have you smoked at least 100 cigarettes in your entire life?" and "Do you now still smoke cigarettes?" Alcohol intake was defined based on self-reported drinking frequency (days per week, month, or year) and drinking quantity (drinks per day; one alcoholic drink is equivalent to 14 $\mathrm{g}$ of pure alcohol) in the past year, and was categorised into four groups: lifetime abstainers $(<12$ drinks in one's life), former drinkers ( $\geq 12$ drinks in a previous year), current light to moderate drinkers ( $\geq 1$ drinks/ week to $\leq 14$ drinks/week for men, or $\geq 1$ drinks/week to $\leq 7$ drinks/week for women), and current heavy drinkers ( $>14$ drinks/week for men or $>7$ drinks/week for women). ${ }^{16}$

\section{Statistical analyses}

We used the $\chi^{2}$ test to assess the baseline characteristics (categorical variables) of the participants across four levels of meeting the physical activity guidelines

Box 1: ICD-10 codes for causes of death used in study

- Cardiovascular disease-100-I09, |11, |13, |20-151, 160-169

- Cancer-C00-C97

- Diabetes-E10-E14

- Chronic lower respiratory tract diseases-J40-J47

- Influenza and pneumonia-J09-18

- Accidents and injuries-V01-X59, Y85-Y86

- Alzheimer's disease-G30

- Nephritis, nephrotic syndrome, or nephrosis-N00N07, N17-N19, N25-N27

ICD-10=international classification of diseases, 10 th revision. (insufficient activity, aerobic only, muscle strengthening only, and both). Cox proportional hazards models were used to calculate the hazard ratios with $95 \%$ confidence intervals to determine the association between levels of meeting the guidelines and all cause and cause specific mortality, with months of follow-up as the time scale. No violations to the proportional hazards assumption were noted using Schoenfeld residual plots. To assess the different potential confounding effects on the association between levels of meeting the guidelines and mortality, we developed three models by sequential inclusion of three groups of covariates into the models. Model 1 included basic personal variables (sex, age, and race/ethnicity), followed by model 2 that additionally included discrete personal (marital status and education level) and lifestyle factors (body mass index, smoking status, and alcohol intake). Model 3 additionally included chronic health conditions (hypertension, heart disease, stroke, cancer, and diabetes). To determine if the associations with mortality differed depending on intensities of aerobic physical activity, we also performed an additional analysis that compared the effects of at least 150 minutes/week of light to moderate aerobic activity versus at least 75 minutes/week of vigorous aerobic activity on all cause and cause specific mortality risk. In addition, interaction terms of potential covariates (age, sex, race/ethnicity, marital status, education level, weight status, smoking status, alcohol intake, and status of chronic health conditions) with level of meeting the physical activity guidelines on mortality outcomes were tested, and we performed subgroup analyses. To test the stability of results, three sensitivity analyses were conducted. Firstly, a meta-analysis was conducted to calculate the summary hazard ratios with 95\% confidence intervals based on results from each of 18 waves (1997-2014) of the NHIS, ${ }^{11}$ with adjustment for potential covariates. Secondly, we performed a sensitivity analysis by excluding participants who died within the first two years of follow-up. Thirdly, a sensitivity analysis was conducted by removing all participants with any chronic conditions at baseline to deal with the possible effect of reverse association. Stratums, clusters, and weights were accounted for in all data analyses for generalisation of the estimates, in consideration of the complex sampling design of the NHIS.

All analyses were conducted using SAS v9.3 (SAS Institute, Cary, NC). A two tailed $\mathrm{P}$ value $<0.05$ was considered to be significant.

\section{Patient and public involvement}

Participants were not involved in setting the research question or the outcome measures, nor were they involved in the design or implementation of the study. No participants were asked to advise on interpretation or writing of the manuscript.

\section{Results}

Population characteristics

Among 479856 eligible adults, 15.9\% ( $\mathrm{n}=76384)$ engaged in both recommended aerobic and muscle 
strengthening activities, 23.7\% ( $\mathrm{n}=113851) \quad$ in recommended aerobic activity only, and 4.5\% $(n=21428)$ in recommended muscle strengthening activity only. The proportion of adults who fully met the 2018 guidelines decreased with age in both men and women (supplementary fig 1). Significant differences in baseline characteristics were found across the four levels of meeting the guidelines (all $\mathrm{P}<0.001$, table 1). Compared with participants who did not meet the guidelines, those who did were more likely to be young, men, white, unmarried, never smokers, and light to moderate alcohol users, and have more than high school education, normal weight, and fewer chronic conditions (table 1).

\section{Recommended physical activity and all cause and} cause specific mortality

During a median follow-up of 8.75 years, 59819 adults died from all causes, 13509 from cardiovascular disease, 14375 from cancer, 3188 from chronic lower respiratory tract diseases, 2477 from accidents and injuries, 1470 from Alzheimer's disease, 1803 from diabetes mellitus, 1135 from influenza and pneumonia, and 1129 from nephritis, nephrotic syndrome, or nephrosis. In the fully adjusted model (model 3), compared with participants not meeting the physical activity guidelines, the risk of all cause mortality was found to be $11 \%$ (hazard ratio 0.89 , $95 \%$ confidence interval 0.85 to 0.94 ) lower in those engaging in recommended muscle strengthening activity and $29 \%(0.71,0.69$ to 0.72$)$ lower in those engaging in recommended aerobic activity, and the risk in those who engaged in both activities was found to be $40 \%(0.60,0.57$ to 0.62$)$ lower (table 2 and fig 1). Similar associations were observed for deaths from cardiovascular disease, cancer, and chronic lower respiratory tract diseases (table 2 and fig 1). In addition, compared with participants who did not meet the physical activity guidelines, the risk of mortality from all eight causes was found to be lower in those who engaged in recommended aerobic activity or in recommended aerobic and muscle strengthening activities, with the most impact found for deaths from chronic lower respiratory tract diseases (aerobic activity: $0.42,0.37$ to 0.47 ; both activities: $0.29,0.23$ to 0.37 ).

An additional analysis was also performed for aerobic activity to compare the effects of at least 150 minutes/week of light to moderate intensity activity with at least 75 minutes/week of vigorous intensity activity on all cause and cause specific mortality risk (supplementary table 1). The effects were found to be largely comparable between the two activities, although the beneficial effect was slightly higher for vigorous than for light to moderate aerobic activity.

\section{Subgroup analyses}

Significant interactions of covariates (age, sex, race/ ethnicity, marital status, education, body mass index category, smoking status, alcohol intake, and chronic conditions) with levels of meeting the physical activity guidelines were found on mortality outcomes (all $\mathrm{P}<0.001)$. In subgroup analyses with stratification by these covariates (table 3), the effect sizes between recommended physical activity and all cause mortality were somewhat stronger among middle aged and older adults (versus young adults), women (versus men), white and black adults (versus Hispanic adults or adults from other racial/ethnic group), adults with high education (versus low education), normal weight adults (versus overweight or obese adults), former smokers (versus never or current smokers), and heavy drinkers (versus never, former, or light to moderate drinkers). Survival benefits in adults with any chronic disease who met the guidelines partly (aerobic) or fully (both aerobic and muscle strengthening) were found to be greater than in those who were healthy. However, similar effect sizes were found for different marital status.

\section{Sensitivity analyses}

Three sensitivity analyses were conducted to confirm the findings. Firstly, the summary hazard ratios (95\% confidence intervals) using meta-analysis of the association with all cause mortality from each survey (1997-2014) was similar to the primary study finding, with 0.90 (0.86 to 0.94 ), 0.68 (0.64 to 0.71 ), and 0.59 ( 0.55 to 0.62 ) for all cause mortality among adults who engaged in muscle strengthening activity only, aerobic activity only, and both activities, respectively. Secondly, the exclusion of participants with chronic conditions at baseline showed little effect on risk estimates for all cause mortality (muscle strengthening: 0.91 (0.83 to 1.00); aerobic: 0.77 (0.74 to 0.80$)$; both activities: 0.66 (0.62 to 0.71$)$ ). Thirdly, the lag analysis, excluding all deaths within the first two years of follow-up, also yielded similar results (muscle strengthening: 0.91 (0.86 to 0.95 ); aerobic: 0.75 (0.73 to 0.77 ); both activities: 0.63 (0.61 to 0.66$)$ ).

\section{Discussion}

Using a large nationally representative sample of US adults, we found that those who engaged in both aerobic and muscle strengthening activities consistent with the recommended 2018 physical activity guidelines for Americans showed a reduced risk of all cause mortality (40\% reduction). Adults who engaged in recommended aerobic or muscle strengthening activities also showed a reduced risk of all cause mortality (29\% and 11\% reduction, respectively). In addition, adults who engaged in recommended aerobic activity were at reduced risk of mortality from all causes and from the eight cause specific outcomes, whereas those who engaged in recommended muscle strengthening activity were found to be at reduced risk of mortality from three causes (cardiovascular disease, cancer, and chronic lower respiratory tract diseases). The study findings provide evidence to support the levels of physical activity recommended in the guidelines for greater survival. Among the 479856 eligible US adults between 1997 and 2014, however, only 15.9\% $(n=76384)$ fully met the guidelines (that is, engaged 


\begin{tabular}{|c|c|c|c|c|c|}
\hline \multirow[b]{2}{*}{ Characteristics } & \multicolumn{4}{|l|}{ Types of physical activity } & \multirow[b]{2}{*}{$P$ values } \\
\hline & $\begin{array}{l}\text { Insufficient aerobic or } \\
\text { muscle strengthening } \\
(n=268 \text { 193) }\end{array}$ & $\begin{array}{l}\text { Muscle } \\
\text { strengthening only } \\
(\mathrm{n}=21428)\end{array}$ & $\begin{array}{l}\text { Aerobic only } \\
(\mathrm{n}=113851)\end{array}$ & $\begin{array}{l}\text { Aerobic and mus- } \\
\text { cle strengthening } \\
(n=76384)\end{array}$ & \\
\hline \multicolumn{6}{|l|}{ Age group (years): } \\
\hline $18-39$ & $89107(35.4)$ & $8181(41.1)$ & $45509(41.4)$ & $40154(53.5)$ & \multirow{3}{*}{$<0.001$} \\
\hline $40-59$ & $94680(37.3)$ & $7422(35.8)$ & $40283(36.9)$ & $25120(33.5)$ & \\
\hline$\geq 60$ & $84406(27.3)$ & $5825(23.2)$ & $28059(21.7)$ & $11110(13.0)$ & \\
\hline \multicolumn{6}{|l|}{ Sex: } \\
\hline Men & $108957(45.1)$ & $10400(52.4)$ & $53885(51.2)$ & $42323(58.3)$ & \multirow{2}{*}{$<0.001$} \\
\hline Women & $159236(54.9)$ & $11028(47.6)$ & $59966(48.8)$ & $34061(41.7)$ & \\
\hline \multicolumn{6}{|l|}{ Race/ethnicity: } \\
\hline Non-Hispanic white & $160319(67.8)$ & $13968(71.2)$ & $77149(74.5)$ & $53217(75.0)$ & \multirow{4}{*}{$<0.001$} \\
\hline Non-Hispanic black & $43705(12.9)$ & $3301(12.8)$ & $13292(9.2)$ & $9464(10.5)$ & \\
\hline Hispanic & $50988(14.2)$ & $3062(10.8)$ & $17223(11.3)$ & $9740(9.8)$ & \\
\hline Other & $13181(5.1)$ & $1097(5.2)$ & $6187(5.1)$ & $3963(4.6)$ & \\
\hline \multicolumn{6}{|l|}{ Education: } \\
\hline$<$ High school & $65683(21.1)$ & $2890(11.7)$ & $15439(12.0)$ & $4930(6.4)$ & \multirow{3}{*}{$<0.001$} \\
\hline High school & $83577(32.3)$ & $5405(25.7)$ & $29866(26.8)$ & $13840(18.5)$ & \\
\hline$>$ High school & $118933(46.6)$ & $13133(62.6)$ & $68546(61.2)$ & $57614(75.1)$ & \\
\hline \multicolumn{6}{|l|}{ Marital status: } \\
\hline Married & $123660(56.6)$ & $9309(53.5)$ & $56221(58.8)$ & $33114(51.9)$ & \multirow{3}{*}{$<0.001$} \\
\hline Divorced/separated/widowed & $80597(20.1)$ & $5994(18.2)$ & $26009(14.5)$ & $14323(11.8)$ & \\
\hline Never married & $63936(23.4)$ & $6125(28.3)$ & $31621(26.7)$ & $28947(36.2)$ & \\
\hline \multicolumn{6}{|l|}{ Body mass index category: } \\
\hline Normal weight & $98149(36.6)$ & $8965(42.1)$ & $46147(40.5)$ & $35474(46.2)$ & \multirow{3}{*}{$<0.001$} \\
\hline Overweight & $91053(34.1)$ & $7608(35.5)$ & $41244(36.3)$ & 27809 (36.5) & \\
\hline Obese & $78991(29.4)$ & 4855 (22.4) & $26460(23.1)$ & $13101(17.3)$ & \\
\hline \multicolumn{6}{|l|}{ Smoking status: } \\
\hline Never & $148613(55.1)$ & $12202(57.8)$ & $63395(55.8)$ & $47346(63.0)$ & \multirow{3}{*}{$<0.001$} \\
\hline Former & $57621(21.7)$ & $5148(23.6)$ & $26645(23.5)$ & $17024(21.9)$ & \\
\hline Current & $61959(23.3)$ & 4078 (18.5) & $23811(20.7)$ & $12014(15.1)$ & \\
\hline \multicolumn{6}{|l|}{ Alcohol intake: } \\
\hline Lifetime abstainer & $75642(26.7)$ & $3729(17.5)$ & $20848(17.7)$ & 8995 (12.5) & \multirow{4}{*}{$<0.001$} \\
\hline Former drinker & $48985(17.4)$ & $3241(13.7)$ & $15426(12.8)$ & $7125(8.8)$ & \\
\hline Light to moderate & $131611(51.4)$ & $13413(64.1)$ & $71030(63.8)$ & $55288(72.5)$ & \\
\hline Heavy & $11955(4.5)$ & $1045(4.8)$ & $6547(5.7)$ & $4976(6.3)$ & \\
\hline \multicolumn{6}{|l|}{ Chronic conditions: } \\
\hline Hypertension & $90644(31.5)$ & $6152(25.9)$ & $29538(24.6)$ & $13761(17.1)$ & $<0.001$ \\
\hline Heart disease & $39574(13.8)$ & $2816(12.1)$ & $11894(10.0)$ & $5904(7.4)$ & $<0.001$ \\
\hline Stroke & $10470(3.5)$ & $698(2.7)$ & $2018(1.6)$ & $760(0.9)$ & $<0.001$ \\
\hline Cancer & $23329(8.3)$ & 1849 (8.1) & $8621(7.4)$ & $4505(5.7)$ & $<0.001$ \\
\hline Diabetes & $28379(9.7)$ & $1680(7.0)$ & 7678 (6.3) & $2703(3.3)$ & $<0.001$ \\
\hline
\end{tabular}

in recommended aerobic and muscle strengthening activities). This highlights that education programmes should include information on the health benefits of physical activity, and well equipped facilities should be developed to facilitate increased physical activity among US adults.

\section{Comparison with other studies}

To our knowledge, only four studies have assessed the combined effects of aerobic and muscle strengthening activities on all cause mortality and cardiovascular disease or cancer specific mortality, with inconsistent findings. ${ }^{7-10}$ Consistent with our findings, data from the NHIS cohort study of 242397 US adults aged 18 years and older (any chronic condition: hazard ratio $0.54,95 \%$ confidence interval 0.48 to 0.61 ; no chronic condition: $0.73,0.60$ to 0.87$){ }^{8}$ the Women's Health Study of 28879 US older women with a mean age of 62.2 years $(0.54,0.47$ to 0.61$),{ }^{7}$ and the Health Survey for England and Scottish Health Survey of 80306 British adults aged 30 years and older $(0.71,0.57 \text { to } 0.87)^{10}$ showed statistically significant survival benefits for all cause mortality among those engaging in recommended aerobic and muscle strengthening activities compared with those who did not. A statistically non-significant association between meeting the guidelines and all cause mortality was, however, reported in data from the National Health and Nutrition Examination Survey that included 10535 participants aged 20 years and older $(0.60,0.35$ to 1.07$) .^{9}$ In addition, a reduced risk of cardiovascular disease specific mortality $(0.43,0.29$ to 0.63$)$ but not cancer specific mortality $(0.93,0.74$ to 1.17) was found among older US women who engaged in both aerobic and muscle strengthening activities. ${ }^{7}$ In contrast, engaging in sufficient levels of aerobic and muscle strengthening activities was associated with significantly reduced cancer specific mortality $(0.70$, 0.50 to 0.98 ) but not cardiovascular disease specific 


\begin{tabular}{|c|c|c|c|c|}
\hline \multirow[b]{2}{*}{ Causes of death } & \multicolumn{4}{|l|}{ Types of physical activity } \\
\hline & $\begin{array}{l}\text { Insufficient aerobic or muscle } \\
\text { strengthening ( } n=268 \text { 193) }\end{array}$ & $\begin{array}{l}\text { Muscle strengthening } \\
\text { only }(n=21428)\end{array}$ & $\begin{array}{l}\text { Aerobic only } \\
(n=113851)\end{array}$ & $\begin{array}{l}\text { Aerobic and muscle } \\
\text { strengthening }(n=76 \text { 384) }\end{array}$ \\
\hline \multicolumn{5}{|l|}{ All causes } \\
\hline No of deaths: & 43798 & 2483 & 10188 & 3350 \\
\hline Model 1 & 1.00 & $0.82(0.78$ to 0.86$)$ & $0.62(0.61$ to 0.64$)$ & $0.48(0.47$ to 0.50$)$ \\
\hline Model 2 & 1.00 & $0.91(0.86$ to 0.95$)$ & $0.69(0.68$ to 0.71$)$ & $0.58(0.56$ to 0.61$)$ \\
\hline Model 3 & 1.00 & $0.89(0.85$ to 0.94$)$ & 0.71 (0.69 to 0.72$)$ & $0.60(0.57$ to 0.62$)$ \\
\hline \multicolumn{5}{|c|}{ Cardiovascular diseases } \\
\hline No of deaths: & 10242 & 539 & 2134 & 594 \\
\hline Model 1 & 1.00 & 0.73 (0.66 to 0.82$)$ & 0.55 (0.52 to 0.58$)$ & $0.38(0.34$ to 0.42$)$ \\
\hline Model 2 & 1.00 & $0.84(0.75$ to 0.94$)$ & $0.62(0.59$ to 0.66$)$ & 0.48 (0.44 to 0.53$)$ \\
\hline Model 3 & 1.00 & $0.82(0.74$ to 0.92$)$ & 0.65 (0.62 to 0.69$)$ & $0.50(0.46$ to 0.56$)$ \\
\hline \multicolumn{5}{|l|}{ Cancer } \\
\hline No of deaths: & 9974 & 593 & 2855 & 953 \\
\hline Model 1 & 1.00 & $0.78(0.71$ to 0.87$)$ & $0.70(0.67$ to 0.73$)$ & $0.50(0.46$ to 0.54$)$ \\
\hline Model 2 & 1.00 & $0.87(0.78$ to 0.96$)$ & $0.76(0.73$ to 0.80$)$ & $0.61(0.56$ to 0.65$)$ \\
\hline Model 3 & 1.00 & $0.85(0.77$ to 0.95$)$ & $0.76(0.73$ to 0.80$)$ & $0.60(0.56$ to 0.65$)$ \\
\hline \multicolumn{5}{|c|}{ Chronic lower respiratory tract diseases } \\
\hline No of deaths: & 2604 & 135 & 349 & 100 \\
\hline Model 1 & 1.00 & $0.65(0.53$ to 0.80$)$ & $0.34(0.30$ to 0.39$)$ & $0.21(0.16$ to 0.26$)$ \\
\hline Model 2 & 1.00 & $0.78(0.63$ to 0.95$)$ & $0.40(0.36$ to 0.46$)$ & $0.29(0.23$ to 0.37$)$ \\
\hline Model 3 & 1.00 & $0.76(0.62$ to 0.93$)$ & $0.42(0.37$ to 0.47$)$ & $0.29(0.23$ to 0.37$)$ \\
\hline \multicolumn{5}{|c|}{ Accidents and injuries } \\
\hline No of deaths: & 1586 & 125 & 525 & 241 \\
\hline Model 1 & 1.00 & $1.00(0.80$ to 1.24$)$ & 0.74 (0.65 to 0.83$)$ & 0.59 (0.50 to 0.70$)$ \\
\hline Model 2 & 1.00 & $1.09(0.88$ to 1.36$)$ & $0.82(0.72$ to 0.93$)$ & $0.70(0.59$ to 0.84$)$ \\
\hline Model 3 & 1.00 & $1.08(0.87$ to 1.35$)$ & $0.82(0.73$ to 0.93$)$ & $0.71(0.60$ to 0.84$)$ \\
\hline \multicolumn{5}{|c|}{ Alzheimer's disease } \\
\hline No of deaths: & 1091 & 63 & 250 & 66 \\
\hline Model 1 & 1.00 & $0.86(0.62$ to 1.18$)$ & $0.71(0.61$ to 0.84$)$ & 0.60 (0.46 to 0.79$)$ \\
\hline Model 2 & 1.00 & $0.90(0.65$ to 1.25$)$ & $0.73(0.62$ to 0.86$)$ & $0.63(0.47$ to 0.84$)$ \\
\hline Model 3 & 1.00 & 0.88 (0.64 to 1.23$)$ & 0.74 (0.62 to 0.87$)$ & $0.64(0.48$ to 0.86$)$ \\
\hline \multicolumn{5}{|l|}{ Diabetes mellitus } \\
\hline No of deaths: & 1407 & 71 & 256 & 69 \\
\hline Model 1 & 1.00 & $0.72(0.55$ to 0.95$)$ & $0.44(0.37$ to 0.51$)$ & $0.26(0.19$ to 0.34$)$ \\
\hline Model 2 & 1.00 & 0.94 (0.71 to 1.24$)$ & 0.57 (0.48 to 0.67$)$ & $0.41(0.31$ to 0.54$)$ \\
\hline Model 3 & 1.00 & 0.96 (0.73 to 1.27$)$ & $0.63(0.53$ to 0.74$)$ & $0.47(0.36$ to 0.62$)$ \\
\hline \multicolumn{5}{|c|}{ Influenza and pneumonia } \\
\hline No of deaths: & 890 & 47 & 151 & 47 \\
\hline Model 1 & 1.00 & $0.77(0.55$ to 1.10$)$ & $0.47(0.38$ to 0.58$)$ & $0.36(0.26$ to 0.50$)$ \\
\hline Model 2 & 1.00 & 0.89 (0.63 to 1.25$)$ & $0.54(0.43$ to 0.67$)$ & $0.45(0.32$ to 0.63$)$ \\
\hline Model 3 & 1.00 & 0.87 (0.62 to 1.24$)$ & 0.55 (0.44 to 0.68$)$ & $0.46(0.32$ to 0.64$)$ \\
\hline \multicolumn{5}{|c|}{ Nephritis, nephrotic syndrome, or nephrosis } \\
\hline No of deaths: & 895 & 44 & 142 & 48 \\
\hline Model 1 & 1.00 & 0.60 (0.42 to 0.86$)$ & $0.38(0.32$ to 0.47$)$ & $0.36(0.25$ to 0.52$)$ \\
\hline Model 2 & 1.00 & 0.72 (0.51 to 1.03$)$ & $0.46(0.37$ to 0.55$)$ & $0.49(0.34$ to 0.71$)$ \\
\hline Model 3 & 1.00 & $0.71(0.50$ to 1.01$)$ & $0.48(0.40$ to 0.59$)$ & $0.52(0.36$ to 0.76$)$ \\
\hline
\end{tabular}

mortality $(0.80,0.55$ to 1.15$)$ in middle aged and older British adults. ${ }^{10}$ However, our findings support the significant association between meeting the physical activity guidelines and mortality due to both cardiovascular disease and cancer. Overall, our study findings substantiate the evidence on the beneficial effects of meeting the guidelines, including the latest 2018 physical activity guidelines for Americans, on reducing the risk of mortality due to all causes and each of the observed eight causes.

The inconsistent findings mentioned might be attributed to differences in how physical activity was measured, the characteristics of the study samples, sample sizes, duration of follow-up, and number and type of confounders included in the models of the different studies. In particular, a previous publication ${ }^{8}$ showed that engaging in recommended muscle strengthening activity only was not associated with reduced all cause mortality, but we found the opposite in our study. Three factors could explain the nonsignificant association in the previous publication. ${ }^{8}$ Firstly, the sample size in that study was about half of that in our study (about 0.24 million versus about 0.48 million). Secondly, the duration of follow-up in that 


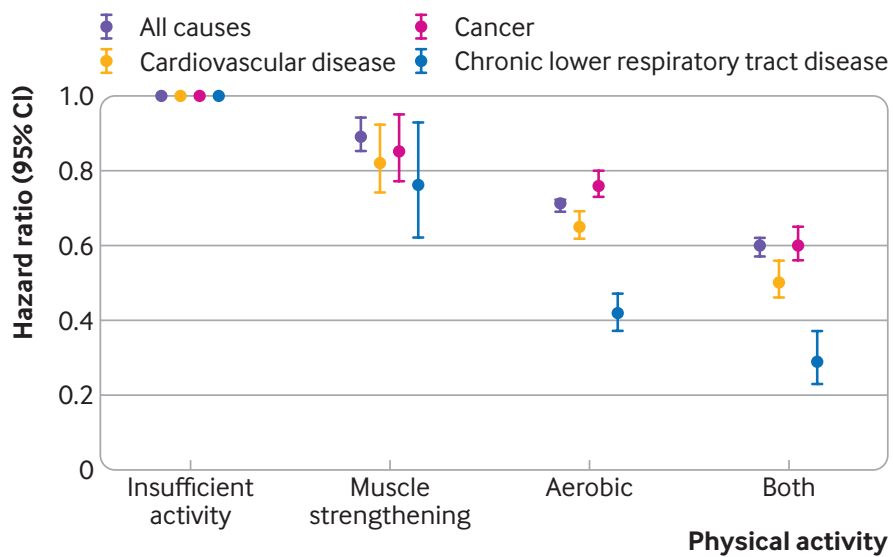

Fig 1 | Association between meeting the 2018 physical activity guidelines for Americans and all cause and cause specific mortality from three diseases. Estimates are from the fully adjusted model that includes the covariates of sex, age, race/ethnicity, education, marital status, body mass index, smoking, alcohol intake, and chronic conditions. Whiskers represent $95 \%$ confidence intervals

study was shorter (5.0 years $v 8.7$ years). Thirdly, the proportion of people meeting the guideline for muscle strengthening activity alone in that study was lower $(3.4 \%(8173 / 242397) v 4.5 \%(21428 / 479856))$. In addition, how physical activity was measured might also explain the differences between studies. In two studies, information on aerobic physical activity was collected using a questionnaire to determine the frequency (times/week) and duration (minutes/time) during the past month before the interview, ${ }^{710}$ whereas in two other studies the timeframe was the past year before interview. ${ }^{89}$ In addition, information on muscle strengthening activity was assessed by both frequency (times/week) and duration (minutes/time) in one of the studies $^{7}$ but by frequency (times/week) in three other studies, ${ }^{8-10}$ as recommended by the physical activity guidelines.

\section{Findings of this study}

In this study, besides the association with mortality due to cardiovascular disease and cancer assessed in previous studies, we also determined whether meeting the physical activity guidelines was associated with mortality due to chronic lower respiratory tract diseases, accidents and injuries, Alzheimer's disease, diabetes mellitus, influenza and pneumonia, and nephritis, nephrotic syndrome, or nephrosis. Our study confirmed that meeting the physical activity guidelines is associated not only with all cause mortality but also with each cause specific mortality. We also found evidence to suggest that the association differs depending on the type of activity followed. For example, engaging only in recommended aerobic physical activity was associated with mortality due to all causes and each of the eight causes, whereas engaging only in recommended muscle strengthening activity was associated with mortality from three main causes only. In addition, engaging in recommended aerobic physical activity tended to provide greater survival benefits than engaging in recommended muscle strengthening activity. Finally, although survival was found to be slightly better for those engaging in at least 75 minutes/week of vigorous physical activity, we also found enhanced survival in those who engaged in at least 150 minutes/week of light to moderate physical activity. Our findings have important public health implications as they suggest that future recommendations could be broadened to include light to moderate physical activity, rather than moderate physical activity only, which will allow for more achievable (yet still beneficial) physical activity goals for the population, especially for those with chronic conditions. Our findings are further supported by a recent meta-analysis, which showed that objectively measured light intensity aerobic physical activity resulted in reduced mortality. ${ }^{4}$

\section{Clinical perspective}

An important observation in our study included greater survival among adults with a diagnosis of chronic conditions who engaged in recommended aerobic physical activity only or in both aerobic and muscle strengthening activities than those who were generally healthy. Given the clearly established connection between physical activity and health, exercise should be viewed as a cost effective way of managing underlying chronic diseases, thereby reducing future mortality risk. ${ }^{17}$ Our finding could have important implications in clinical practice as it suggests that inclusion of recommended aerobic and muscle strengthening activities or recommended aerobic physical activity alone into chronic disease management of US adults is possible and has survival benefits. Our findings support the recommendations of the 2018 physical activity guidelines for Americans that adults with chronic conditions and physical capability should also perform at least 150 minutes a week of moderate intensity aerobic activity or 75 minutes a week of vigorous intensity aerobic activity, as well as muscle strengthening activity for two days a week-the same recommendations as those for adults without any chronic conditions. ${ }^{18}$ Lack of time is, however, one important barrier for people to meet the guidelines. For people who are unwilling or are unable to do both aerobic and muscle strengthening exercises, aerobic physical activity could be prioritised over muscle strengthening activity given the greater health benefit shown in our study. Moreover, diagnoses of chronic conditions are another barrier for adults to engage in recommended levels of physical activity. ${ }^{19}$ For adults with such conditions who are unable to meet the physical activity guidelines, appropriate types, intensity, and amounts of physical activity could be adapted based on abilities and the severity of the chronic conditions. ${ }^{18}$ As we observed that engaging in at least 150 minutes a week of light to moderate physical activity was largely comparable with at least 75 minutes a week of vigorous physical activity based on our studied outcomes, the former could be prioritised in those people where light to moderate intensity exercise is desired or more achievable, such as those with severe chronic disease. 


\begin{tabular}{|c|c|c|c|c|}
\hline \multirow[b]{2}{*}{ Subgroups } & \multicolumn{4}{|l|}{ Types of physical activity } \\
\hline & $\begin{array}{l}\text { Insufficient aerobic or } \\
\text { muscle strengthening }\end{array}$ & $\begin{array}{l}\text { Muscle strengthening } \\
\text { only }\end{array}$ & Aerobic only & $\begin{array}{l}\text { Aerobic and muscle } \\
\text { strengthening }\end{array}$ \\
\hline \multicolumn{5}{|l|}{ Age group (years): } \\
\hline $18-39$ & 1.00 & $0.95(0.78$ to 1.15$)$ & 0.79 (0.71 to 0.87$)$ & $0.71(0.63$ to 0.79$)$ \\
\hline $40-59$ & 1.00 & $0.95(0.85$ to 1.06$)$ & $0.76(0.72$ to 0.80$)$ & $0.61(0.56$ to 0.67$)$ \\
\hline$\geq 60$ & 1.00 & 0.87 (0.82 to 0.92$)$ & 0.69 (0.67 to 0.71$)$ & $0.58(0.55$ to 0.61$)$ \\
\hline \multicolumn{5}{|l|}{ Sex: } \\
\hline Men & 1.00 & 0.90 (0.84 to 0.97$)$ & $0.72(0.70$ to 0.74$)$ & 0.61 (0.58 to 0.64$)$ \\
\hline Women & 1.00 & 0.89 (0.83 to 0.95$)$ & 0.69 (0.67 to 0.72$)$ & 0.57 (0.53 to 0.61$)$ \\
\hline \multicolumn{5}{|l|}{ Race/ethnicity: } \\
\hline Non-Hispanic white & 1.00 & 0.89 (0.84 to 0.94$)$ & 0.70 (0.68 to 0.71$)$ & 0.59 (0.56 to 0.61$)$ \\
\hline Non-Hispanic black & 1.00 & $0.92(0.80$ to 1.06$)$ & 0.75 (0.69 to 0.81$)$ & 0.61 (0.53 to 0.70$)$ \\
\hline Hispanic & 1.00 & $0.94(0.77$ to 1.13$)$ & 0.83 (0.75 to 0.90$)$ & 0.70 (0.60 to 0.81$)$ \\
\hline Other & 1.00 & 0.80 (0.60 to 1.06$)$ & 0.78 (0.67 to 0.92$)$ & 0.82 (0.66 to 1.03$)$ \\
\hline \multicolumn{5}{|l|}{ Education: } \\
\hline$<$ High school & 1.00 & $0.86(0.78$ to 0.95$)$ & $0.72(0.68$ to 0.75$)$ & $0.64(0.57$ to 0.70$)$ \\
\hline High school & 1.00 & 0.90 (0.83 to 0.99$)$ & $0.71(0.68$ to 0.74$)$ & 0.63 (0.58 to 0.68$)$ \\
\hline$>$ High school & 1.00 & 0.91 (0.84 to 0.98$)$ & 0.71 (0.68 to 0.73$)$ & $0.60(0.57$ to 0.63$)$ \\
\hline \multicolumn{5}{|l|}{ Marital status: } \\
\hline Married & 1.00 & 0.87 (0.81 to 0.94$)$ & 0.71 (0.69 to 0.73$)$ & $0.60(0.57$ to 0.64$)$ \\
\hline Divorced/separated/widowed & 1.00 & 0.89 (0.84 to 0.94$)$ & 0.69 (0.66 to 0.72$)$ & $0.58(0.54$ to 0.61$)$ \\
\hline Never married & 1.00 & 0.94 (0.81 to 1.10$)$ & 0.75 (0.69 to 0.81$)$ & 0.59 (0.53 to 0.67$)$ \\
\hline \multicolumn{5}{|l|}{ Body mass index category: } \\
\hline Normal weight & 1.00 & $0.86(0.79$ to 0.92$)$ & 0.70 (0.67 to 0.72$)$ & 0.53 (0.50 to 0.57$)$ \\
\hline Overweight & 1.00 & 0.93 (0.86 to 1.01$)$ & 0.71 (0.68 to 0.74$)$ & 0.64 (0.60 to 0.68$)$ \\
\hline Obese & 1.00 & 0.91 (0.82 to 1.02$)$ & $0.73(0.69$ to 0.78$)$ & 0.69 (0.63 to 0.76$)$ \\
\hline \multicolumn{5}{|l|}{ Smoking status: } \\
\hline Never & 1.00 & 0.95 (0.88 to 1.02$)$ & $0.72(0.69$ to 0.75$)$ & $0.64(0.60$ to 0.68$)$ \\
\hline Former & 1.00 & 0.87 (0.81 to 0.94$)$ & 0.67 (0.65 to 0.70$)$ & 0.55 (0.51 to 0.58$)$ \\
\hline Current & 1.00 & $0.85(0.75$ to 0.96$)$ & 0.77 (0.73 to 0.81$)$ & $0.66(0.60$ to 0.73$)$ \\
\hline \multicolumn{5}{|l|}{ Alcohol intake: } \\
\hline Lifetime abstainer & 1.00 & $1.04(0.93$ to 1.16$)$ & $0.72(0.68$ to 0.76$)$ & 0.62 (0.56 to 0.69$)$ \\
\hline Former drinker & 1.00 & $0.90(0.82$ to 0.99$)$ & 0.65 (0.62 to 0.68$)$ & $0.62(0.57$ to 0.68$)$ \\
\hline Light to moderate & 1.00 & $0.87(0.81$ to 0.94$)$ & $0.74(0.71$ to 0.76$)$ & $0.62(0.59$ to 0.66$)$ \\
\hline Heavy & 1.00 & $0.70(0.55$ to 0.89$)$ & 0.75 (0.67 to 0.84$)$ & $0.51(0.42$ to 0.61$)$ \\
\hline \multicolumn{5}{|l|}{ Chronic conditions: } \\
\hline None & 1.00 & $0.91(0.83$ to 1.00$)$ & 0.77 (0.74 to 0.80$)$ & $0.66(0.62$ to 0.71$)$ \\
\hline Any chronic conditions & 1.00 & $0.90(0.85$ to 0.95$)$ & 0.66 (0.65 to 0.68$)$ & $0.55(0.52$ to 0.58$)$ \\
\hline Hypertension & 1.00 & 0.91 (0.86 to 0.97$)$ & 0.69 (0.67 to 0.72$)$ & $0.59(0.55$ to 0.63$)$ \\
\hline Heart disease & 1.00 & $0.90(0.83$ to 0.98$)$ & $0.66(0.63$ to 0.70$)$ & $0.56(0.52$ to 0.60$)$ \\
\hline Stroke & 1.00 & $0.91(0.79$ to 1.06$)$ & $0.65(0.59$ to 0.71$)$ & $0.74(0.64$ to 0.86$)$ \\
\hline Cancer & 1.00 & $0.87(0.79$ to 0.96$)$ & $0.63(0.59$ to 0.67$)$ & $0.51(0.46$ to 0.56$)$ \\
\hline Diabetes & 1.00 & 0.98 (0.87 to 1.10$)$ & 0.68 (0.64 to 0.72$)$ & 0.60 (0.53 to 0.67$)$ \\
\hline
\end{tabular}

\section{Strengths and limitations of this study}

The study has important strengths. Firstly, the study was a prospective cohort study based on data from a large $(n=479856)$ nationally representative survey among US adults. Secondly, as measures of several potential confounding factors (personal, lifestyle, chronic conditions) were available at each survey using consistent methodology, ${ }^{11}$ we were able to adjust for these in our models. Thirdly, several sensitivity analyses were performed to confirm our findings.

Despite these strengths, the study has several limitations. Firstly, the measurements of both aerobic and muscle strengthening activities were self-reported by participants and that might have resulted in recall bias, compared with exposure assessment measured using objective means such as the accelerometer, which can provide a more accurate assessment of the true level of physical activity. However, misclassification of exposure status by a self-reported method usually results in regression dilution bias and an underestimate of the magnitude of the true association. ${ }^{10}$ Secondly, the assessment of muscle strengthening activity was based on frequency only as the duration of sessions was not available; however, this definition is consistent with the 2018 physical activity guidelines for Americans, which recommend meeting sufficient frequency $(\geq 2$ times/week) regardless of duration. Thirdly, aerobic physical activity in the study was only measured during leisure time. Occupational, transport, and household related physical activity, which have been shown to reduce mortality risk, ${ }^{20}$ were not collected in the NHIS surveys. Not considering the domains of physical activity might underestimate the benefits of total physical activity. Fourthly, information on the severity of reported chronic conditions was not available. Our results should be interpreted with caution as it is likely 
that these conditions might be less severe in most participants in the study sample who were able to meet the guidelines. Fifthly, 49507 of 529363 participants $(9.35 \%)$ were excluded owing to missing data on exposures or covariates, as well as being pregnant, which might have led to bias if those excluded differed from those not excluded. Sixthly, as per the guidelines and other previous versions for adults who require aerobic physical activity to occur in bouts of at least 10 minutes, frequency of aerobic physical activity was assessed in the NHIS 1997-2014 as the number of times participants engaged in an exercise that lasted at least 10 minutes. An important change in the 2018 guidelines is that aerobic physical activity can be accumulated in bouts of less than 10 minutes-now considered enough to promote increases in health enhancing physical activity. Further prospective cohort studies are necessary to determine the association between aerobic physical activity not required to last at least 10 minutes and mortality. Furthermore, since our findings were based on nationally representative adults in the US, the generalisability to populations of other races and ethnicities outsides the US should be made with caution. Additionally, the 2018 guidelines recommend that it is important to work all the major muscle groups of the body-the legs, hips, back, abdomen, chest, shoulders, and arms. However, the question in the NHIS survey (1997-2014) used to collect data on the frequency of muscle strengthening activity only highlighted lifting weights or doing calisthenics. It is possible that this definition, used in our study, might have misclassified some participants, which could have contributed to the weaker association between muscle strengthening activity and mortality. Another limitation would be that physical activity was measured at a single point at baseline in our study, and exercise patterns could have changed over time, which might have influenced the association. Future prospective cohort studies with serial measures are needed to determine how maintenance in meeting guideline physical activity levels, or change in status, relate to health outcomes. Finally, although in our study we considered at least 10 potential confounding factors, including personal variables, lifestyle factors, and history of chronic conditions, residual and unmeasured confounding might have influenced the association. In addition, the sensitivity analyses (for example, by excluding those who died in the first few years or those with chronic conditions at baseline) could have partly, rather than totally, overcome reverse causation.

\section{Conclusions}

Engaging in leisure time aerobic physical activity and muscle strengthening activity at levels recommended by the 2018 physical activity guidelines was statistically significantly associated with reduced risk of all cause and cause specific mortality. Engaging in recommended aerobic physical activity was associated with reduced risk of mortality from all eight causes examined, whereas engaging in muscle strengthening activity was associated with reduced risk of mortality from three causes, including cardiovascular disease, cancer, and chronic lower respiratory tract diseases. In addition, greater survival benefits in adults with chronic conditions were associated with meeting the physical activity guidelines. Our findings support that the physical activity levels recommended in the 2018 physical activity guidelines for Americans provide important survival benefits. Additionally, in accordance with the guidelines, more physical activity than the minimum recommendation could provide greater health benefits.

\section{AUTHOR AFFILIATIONS}

${ }^{1}$ Department of Nutrition and Food Hygiene, School of Public Health, Cheeloo College of Medicine, Shandong University, Jinan, China

${ }^{2}$ The Key Laboratory of Cardiovascular Remodeling and Function Research, Chinese Ministry of Education, Chinese National Health Commission and Chinese Academy of Medical Sciences, The State and Shandong Province Joint Key Laboratory of Translational Cardiovascular Medicine, Department of Cardiology, Qilu Hospital, Cheeloo College of Medicine, Shandong University, Jinan, China

${ }^{3}$ Precision Health Economics and Outcomes Research, Los Angeles, CA, USA

${ }^{4}$ Department of Preventive Medicine and Community Health, University of Texas Medical Branch, Galveston, TX, USA

${ }^{5}$ Menzies Institute for Medical Research, University of Tasmania, 7000 Hobart, Australia

${ }^{6}$ Research Centre of Applied and Preventive Cardiovascular Medicine, University of Turku, Turku, Finland

${ }^{7}$ Centre for Population Health Research, University of Turku and Turku University Hospital, Turku, Finland

${ }^{8}$ Department of Epidemiology, School of Public Health, Cheeloo College of Medicine, Shandong University, Jinan250012, China

We thank the National Center for Health Statistics of the Centers for Disease Control and Prevention for sharing the National Health Interview Survey data and Jiahong Sun and Liu Yang from Shandong University for improving the English in the manuscript.

Contributors: BX designed the study and is the principal investigator MZ conducted the data analysis and drafted the manuscript. SPV, CGM, and BX critically revised the manuscript for important intellectual content. All authors approved the final version of the manuscript. BX is guarantor. The corresponding author attests that all the listed authors meet the authorship criteria and that no others meeting the criteria have been omitted.

Funding: BX was supported by the Innovation Team of "Climbing" Program of Shandong University, and Youth Team of Humanistic and Social Science of Shandong University (20820IFYT1902). CGM was supported by a National Heart Foundation of Australia future leader fellowship (100849). The funders had no role in the study design or implementation; data collection, management, analysis, or interpretation; manuscript preparation, review, or approval; or the decision to submit the manuscript for publication.

Competing interests: All authors have completed the ICMIE uniform disclosure form at www.icmje.org/coi_disclosure.pdf and declare: support from the Innovation Team of "Climbing" Program of Shandong University, Youth Team of Humanistic and Social Science of Shandong University, and National Heart Foundation of Australia future leader fellowship for the submitted work; no financial relationships with any organisations that might have an interest in the submitted work in the previous three years; no other relationships or activities that could appear to have influenced the submitted work.

Ethical approval: The National Health Interview Survey data are deidentified and do not include any protected health information. The data are publicly available and exempt under the ethical board review of the corresponding author's institution.

Data sharing: The National Health Interview Survey data are available at www.cdc.gov/nchs/nhis/index.htm

Dissemination to participants and related patient and public communities: The results of the research will be disseminated to the public through broadcasts, popular science articles, and newspapers. The study guarantor (BX) affirms that the manuscript is an honest, accurate, and transparent account of the study being reported; that 
no important aspects of the study have been omitted; and that any discrepancies from the study as planned have been explained.

This is an Open Access article distributed in accordance with the Creative Commons Attribution Non Commercial (CC BY-NC 4.0) license, which permits others to distribute, remix, adapt, build upon this work non-commercially, and license their derivative works on different terms, provided the original work is properly cited and the use is noncommercial. See: http://creativecommons.org/licenses/by-nc/4.0/.

1 Lee IM, Shiroma EJ, Lobelo F, Puska P, Blair SN, Katzmarzyk PT, Lance Physical Activity Series Working Group. Effect of physical inactivity on major non-communicable diseases worldwide: an analysis of burden of disease and life expectancy. Lancet 2012;380:219-29. doi:10.1016/S0140-6736(12)61031-9

2 Ding D, Lawson KD, Kolbe-Alexander TL, et al, Lancet Physical Activity Series 2 Executive Committee. The economic burden of physical inactivity: a global analysis of major non-communicable diseases. Lancet 2016:388:1311-24. doi:10.1016/S0140-6736(16)30383-X

$3 \mathrm{Kyu} \mathrm{HH}$, Bachman VF, Alexander LT, et al. Physical activity and risk of breast cancer, colon cancer, diabetes, ischemic heart disease, and ischemic stroke events: systematic review and dose-response meta-analysis for the Global Burden of Disease Study 2013. BMJ 2016;354:i3857. doi:10.1136/bmj.i3857

4 Ekelund U, Tarp J, Steene-Johannessen J, et al. Dose-response associations between accelerometry measured physical activity and sedentary time and all cause mortality: systematic review and harmonised meta-analysis. BMJ 2019;366:14570. doi:10.1136/bmj. 14570

5 US Department of Health and Human Services. Physical Activity Guidelines for Americans. 2nd ed. US Department of Health and Human Services, 2018.

6 US Department of Health and Human Services. 2008 Physical Activity Guidelines for Americans. US Department of Health and Human Services, 2008.

7 Kamada M, Shiroma EJ, Buring JE, Miyachi M, Lee IM. Strength Training and All-Cause, Cardiovascular Disease, and Cancer Mortality in Older Women: A Cohort Study. J Am Heart Assoc 2017;6:e007677. doi:10.1161/JAHA.117.007677

8 Schoenborn CA, Stommel M. Adherence to the 2008 adult physical activity guidelines and mortality risk. Am J Prev Med 2011;40:51421. doi:10.1016/i.amepre.2010.12.029.

9 Zhao G, Li C, Ford ES, et al. Leisure-time aerobic physical activity, muscle-strengthening activity and mortality risks among US adults: the NHANES linked mortality study. Br J Sports Med 2014:48:244-9. doi:10.1136/bjsports-2013-092731
10 Stamatakis E, Lee IM, Bennie J, et al. Does Strength-Promoting Exercise Confer Unique Health Benefits? A Pooled Analysis of Data on 11 Population Cohorts With All-Cause, Cancer, and Cardiovascular Mortality Endpoints. Am J Epidemiol 2018;187:1102-12. doi:10.1093/aje/kw×345

11 Centers for Disease Control and Prevention. www.cdc.gov/nchs/nhis/ index.htm

12 National Center for Health Statistics, Centers for Disease Control and Prevention. The Linkage of National Center for Health Statistics Survey Data to the National Death Index-2015 Linked Mortality File (LMF): Methodology Overview and Analytic Considerations. www. cdc.gov/nchs/data/datalinkage/Imf2015_methodology_analytic_ considerations.pdf.

13 National Center for Health Statistics. NHANES I Epidemiologic Follow-up Survey (NHEFS)-Calibration Sample for NDI Matching Methodology. www.cdc.gov/nchs/data/datalinkage/mort calibration_study.pdf.

14 Lochner K, Hummer RA, Bartee S, Wheatcroft G, Cox C. The public-use National Health Interview Survey linked mortality files: methods of reidentification risk avoidance and comparative analysis. Am J Epidemiol 2008;168:336-44. doi:10.1093/aje/kwn123

15 World Health Organization. International Statistical Classification of Diseases and Related Health Problems. 10th Revision. 2015. https:// icd.who.int/browse10/2015/en.

16 Xi B, Veeranki SP, Zhao M, Ma C, Yan Y, Mi J. Relationship of Alcohol Consumption to All-Cause, Cardiovascular, and Cancer-Related Mortality in U.S. Adults. J Am Coll Cardiol 2017;70:913-22. doi:10.1016/j.jacc.2017.06.054

17 Sallis R, Franklin B, Joy L, Ross R, Sabgir D, Stone J. Strategies for promoting physical activity in clinical practice. Prog CardiovasC Dis 2015;57:375-86. doi:10.1016/j.pcad.2014.10.003

18 Piercy KL, Troiano RP, Ballard RM, et al. The Physical Activity Guidelines for Americans. JAMA 2018:320:2020-8 doi:10.1001/ jama.2018.14854

19 Petter M, Blanchard C, Kemp KAR, Mazoff AS, Ferrier SN. Correlates of exercise among coronary heart disease patients: review, implications and future directions. Eur / Cardiovasc Prev Rehabil 2009;16:51526. doi:10.1097/HJR.0b013e3283299585

20 Autenrieth CS, Baumert J, Baumeister SE, et al. Association between domains of physical activity and all-cause, cardiovascular and cancer mortality. Eur J Epidemiol 2011;26:91-9. doi:10.1007/s10654-010 $9517-6$

Supplementary information: additional figure and table 